\title{
Polyester fabric modification through fabrication of nano-copper compounds
}

\section{Modificación de tejido de poliéster mediante la fabricación de}

compuestos de nano-cobre.

Author:

Mohammad Mahdi Jolae ${ }^{1}$

Majid Montazer ${ }^{2 *}$

Abo-Saeed Rashidi ${ }^{1}$

Mohammad Bameni Moghadam ${ }^{3}$

\section{SCIENTIFIC RESEARCH}

How to cite this paper:

Jolaei M., Montazer M ., Rashidi A ., Bameni Moghadam M., Polyester fabric modification through fabrication of nano copper compound, Iran. Innovaciencia 2020; 8 (1): 1-12. DOI:http://dx.doi.org/10.15649 $\underline{\angle 2346075 X .1005}$

Reception date:

Received: 30 May 2020

Accepted: 30 September 2020

Published: 1 December 2020

Keywords:

In situ synthesis, nano-plates copper, polyester fabric, cetyltrimethylammonium bromide (CTAB)

\section{ABSTRACT}

Introduction This research introduces a new method of polyester fabric surface modification in order to achieve distinctive features. Materials and methods. The copper sulfate, sodium hydroxide and cetyl trimethylammonium bromide (CTAB) were used to synthesize copper nanoparticles, and loaded on the polyester fabric surface. The optimal sample was considered by SEM-EDX, FT-IR and XRD devices. Results SEM images showed copper nanoparticles in shape of nano-plates with 150 to 600 $\mathrm{nm}$ in length and thickness of about $30 \mathrm{~nm}$. The best results obtained on the modified fabric processed at boil for $120 \mathrm{~min}$. According to the results of experiments $\mathrm{CuO}$ and $\mathrm{Cu}^{0}$ were synthesized on the fabric.

1 Department of Textile Engineering, Science and Research Branch, Islamic Azad University, Tehran, Iran.

$2^{*}$ Department of Textile Engineering, Amirkabir University of Technology, Tehran, Iran. Corresponding 


\section{INTRODUCTION}

At the moment, attention to the nanomaterials is very important due to the low production costs and novel properties in the nanoscale. The latest research indicates an increased anticancer effect of nanomaterials such as gold, silver, and copper nanoparticles [1].

The synthesis of copper nanoparticles has become very important for its inherent properties. One of the most important methods available for the synthesis of copper nanoparticles is the chemical reduction method [2] to [4]. The desire to use copper nanoparticles has increased due to new optical, catalytic, mechanical, electrical, magnetic and thermal conductivity properties compared to gold nanoparticles and silver nanoparticles [5].

Several methods have been proposed to improve the structure of the fabric, which can generally be classified into two categories of chemical and physical methods. The physical methods are: plasma, corona, laser, electron beam, ion and neutron and chemical methods are: surface grafting, sol gel, use of different materials and microcapsules [6].

Polyester fabric was modified by synthesizing copper nanoparticles using triethanolamine as polyester aminolysis and $\mathrm{pH}$ adjustment, copper sulfate as a metal salt, sodium hypochosphite as reducing agent, and polyvinyl pyrrolidone as stabilizer. The modified fabrics indicated high tensile strength and flame retardant properties [7].

Acetonitrile has been used for the first time as a protective agent for the preparation of copper nanoparticles. Copper nanoparticles usually have a cube-shaped form with diameters less than 100 nanometers [8]. A number of researchers worked on the synthesis of nano-sized copper through the colloidal solution and its application on cotton fabrics. Nano-colloid of copper by chemical reduction of copper salt using sodium borohydride as a reducing agent is used in the presence of trisodium citrate produced particles with the average particle size of 60 to $100 \mathrm{~nm}$ [9]. Also we investigated the usage of alkaline glucose for synthesis of copper nanoparticles on polyester fabric forming nano-copper with a thickness of $30-40 \mathrm{~nm}$ on the surface of the fabric [10].

Also, the modification of the polyester fabric with betacyclodextrin has been reported with citric acid, butane tetracarboxylic acid, dimethyl dihydroxyl ethylene urea, and two siloxane-based softeners as a crosslinking agent.
They showed the higher durability of beta-cyclodextrin on the polyester after 10 washes by using cross-linking agents [10] to [13].

Further, the antibacterial activities of copper nanoparticles were studied and confirmed their usage as antibacterial agent [14] to [15].

In this study, the surface modification of polyester fabric was performed and three factors of weight changes, color changes and antibacterial properties were considered and the sample with the highest weight and least color change was selected as the optimal sample. In addition, the antibacterial property was reported excellent for all samples due to the complete elimination of bacteria.

Applicability, availability and simplicity of methods are some of the most important features of this study.

\section{MATERIALS}

Materials used in this research were: polyester fabrics with $165 \mathrm{~g} / \mathrm{m}^{2}$ from the local market (Iran) and copper sulfate $\left(\mathrm{CuSO}_{4} * 5 \mathrm{H}_{2} \mathrm{O}\right)$, sodium hydroxide $(\mathrm{NaOH})$ and cetyltrimethylammonium bromide (CTAB) from Merck Co.(Germany).

\section{INSTRUMENTS}

The absorption rate of nano-copper was studied by a spectrometer called Varian, Cary100 UV-Vis-NIR. Reflection spectra of nano-copper on the fabrics were investigated by a Spectrophotometer Color-Eye 7000A (USA). Surface morphology of the fabric was observed by scanning electron microscope TESCAN VEGA $5130 \mathrm{~mm}$ SEM/EDX (Czech Republic). To determine chemical groups on the fabric, and FT-IR spectrometer (model Bomem-mb100) was (Canada) used. Also, the crystal structure of the fabric was investigated by an X-Ray diffraction system (model XRD PTS 300) from SEIFERT Co. (Germany).

\section{EXPERIMENTS}

The design of the experiments was based on the response surface methodology (RSM) and using the Central Composite Design method (CCD). According to the design, 25 samples of polyester fabric in the size of $10 *$ $20 \mathrm{~cm}^{2}$ were prepared and then washed with a nonionic detergent $(1 \mathrm{~g} / \mathrm{L})$ at $60^{\circ} \mathrm{C}$ for $20 \mathrm{~min}$. Finally, the samples were dried and cured. The experiments were carried out at the boiling point (Table 1). 
Table 1. Different factors based on response surface methodology (RSM) analysis and weight and color changes

\begin{tabular}{|c|c|c|c|c|c|c|}
\hline NO & Copper sulfate (w/v \%) & $\mathrm{NaOH}(\mathrm{w} / \mathrm{v} \%)$ & CTAB (w/v \%) & Time (min) & $\mathrm{W}(\%)$ & $\Delta \mathrm{E}$ \\
\hline 1 & 1 & 0.29 & 1 & 77.42 & -3.20 & 46.33 \\
\hline 2 & 1 & 0.1 & 0.35 & 120 & 4.11 & 13.52 \\
\hline 3 & 1 & 1 & 1 & 120 & -2.88 & 19.70 \\
\hline 4 & 0.1 & 1 & 0.01 & 45 & -3.07 & 39.42 \\
\hline 5 & 1 & 0.1 & 0.01 & 45 & -2.94 & 21.45 \\
\hline 6 & 0.1 & 1 & 0.01 & 120 & -2.05 & 35.55 \\
\hline 7 & 1 & 1 & 1 & 45 & -2.16 & 29.81 \\
\hline 8 & 0.1 & 0.1 & 1 & 45 & 2.99 & 18.85 \\
\hline 9 & 0.41 & 0.1 & 1 & 120 & -3.35 & 17.54 \\
\hline 10 & 0.1 & 0.51 & 0.64 & 120 & -1.00 & 6.27 \\
\hline 11 & 1 & 1 & 1 & 45 & -3.77 & 21.75 \\
\hline 12 & 0.67 & 0.51 & 0.01 & 120 & -1.90 & 9.81 \\
\hline 13 & 0.55 & 1 & 0.5 & 81.94 & -2.94 & 15.64 \\
\hline 14 & 0.78 & 0.54 & 0.52 & 45 & 2.17 & 24.66 \\
\hline 15 & 0.97 & 0.96 & 0.33 & 120 & 2.53 & 29.49 \\
\hline 16 & 1 & 0.1 & 0.01 & 45 & -3.76 & 8.10 \\
\hline 17 & 0.1 & 1 & 0.01 & 120 & -0.65 & 9.72 \\
\hline 18 & 0.96 & 0.1 & 0.97 & 120 & -4.11 & 9.28 \\
\hline 19 & 0.1 & 0.1 & 0.01 & 88.92 & -3.62 & 44.78 \\
\hline 20 & 0.55 & 0.31 & 0.48 & 89.04 & 4.26 & 10.68 \\
\hline 21 & 0.67 & 0.51 & 0.01 & 120 & 3.60 & 12.31 \\
\hline 22 & 0.13 & 0.39 & 0.33 & 45 & -1.79 & 20.21 \\
\hline 23 & 0.1 & 1 & 1 & 75.69 & -3.84 & 23.14 \\
\hline 24 & 0.1 & 0.1 & 1 & 45 & -3.34 & 18.85 \\
\hline 25 & 1 & 1 & 0.01 & 75.54 & 2.59 & 13.54 \\
\hline
\end{tabular}

In the first step, weight changes, color changes and antibacterial properties were calculated for different samples and the optimum specimen was determined.

In terms of the values obtained from color changes and weight changes using the Design-Expert software (DOE), related models and equations were obtained.

Figure (1) and (2) show the three-dimensional response level for weight variations and color variations. 


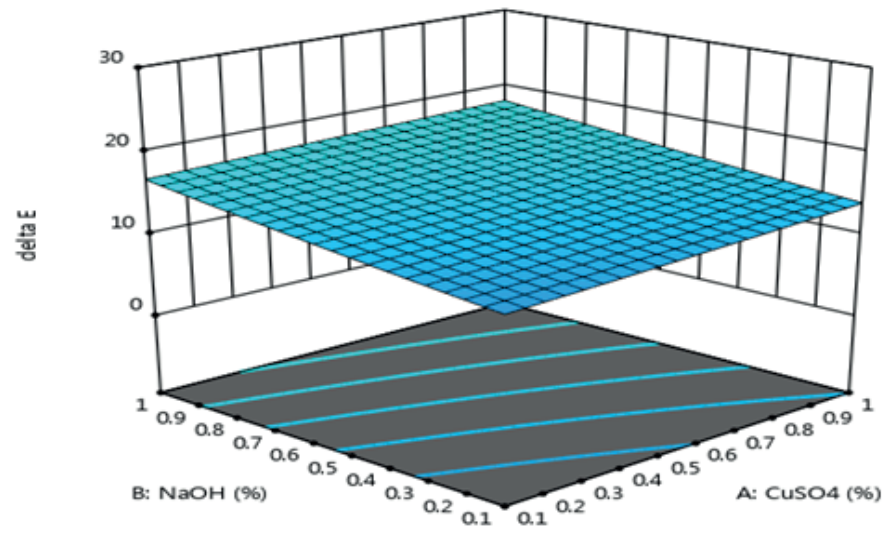

Fig1. 3D response surface for color variations

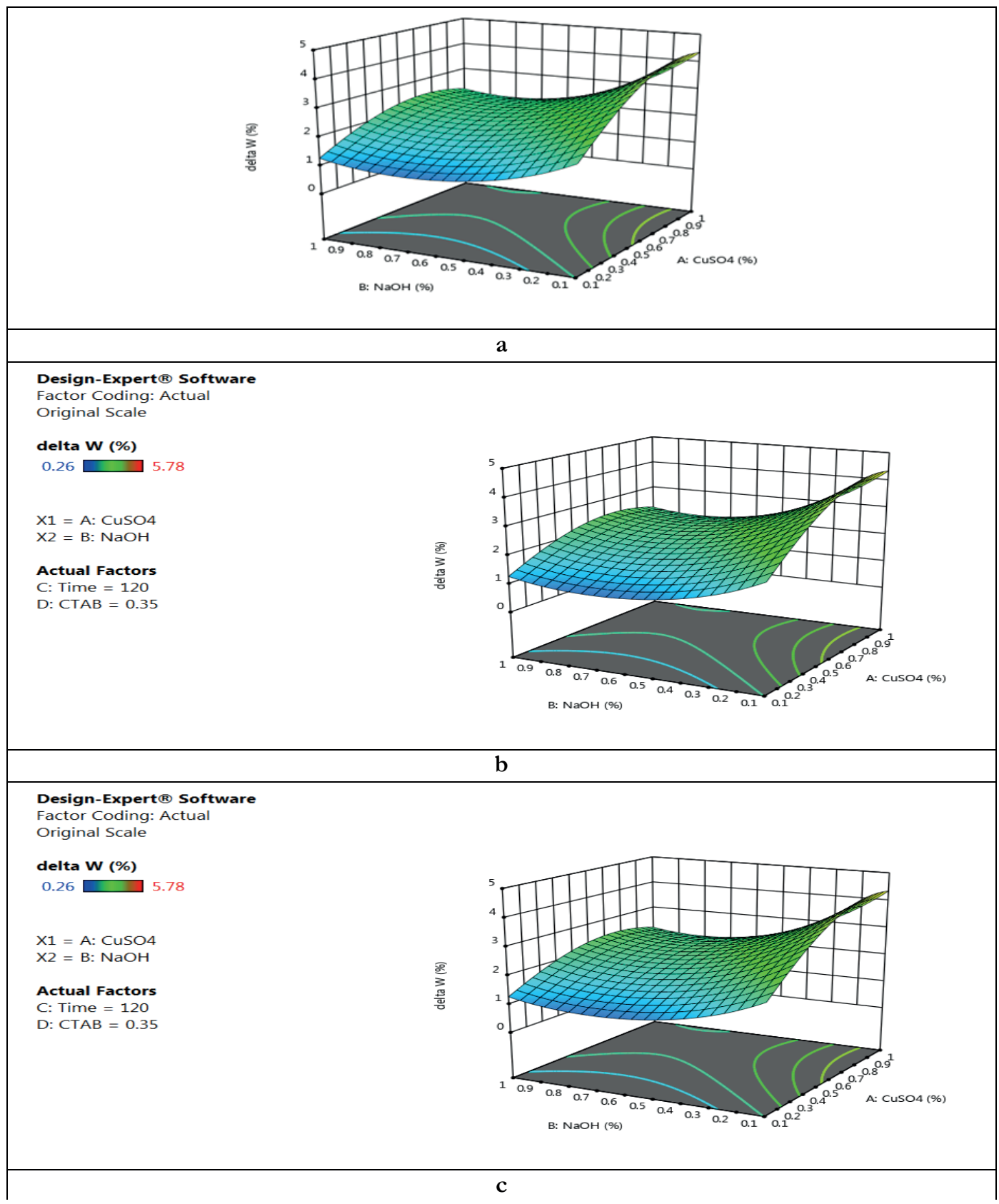


Design-Expert( $\otimes^{\circledR}$ Software

Factor Coding: Actual

Original Scale

delta $\mathbf{W}(\%)$

$0.26 \square 5.78$

$\mathrm{x} 1=\mathrm{A}: \mathrm{CuSO} 4$

$\mathrm{X} 2=\mathrm{B}: \mathrm{NaOH}$

Actual Factors

C: Time $=120$

$\mathrm{D}: \mathrm{CTAB}=0.35$

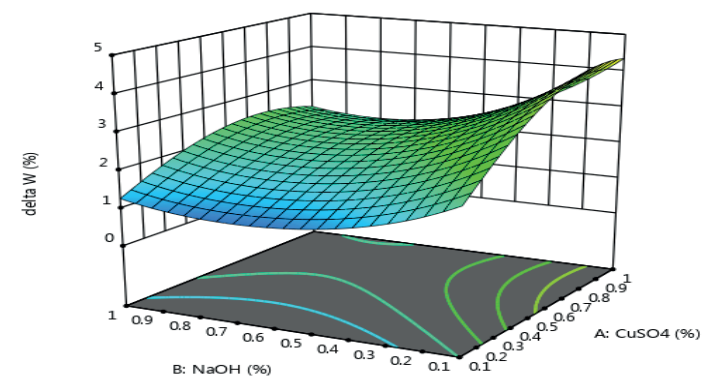

d

Design-Expert (®) Software

Factor Coding: Actual

Original Scale

delta $\mathbf{w}(\%)$

$0.26 \square 5.78$

$\mathrm{X} 1=\mathrm{A}: \mathrm{CuSO} 4$

$\mathrm{X}_{2}=\mathrm{B}: \mathrm{NaOH}$

Actual Factors

C: Time $=120$

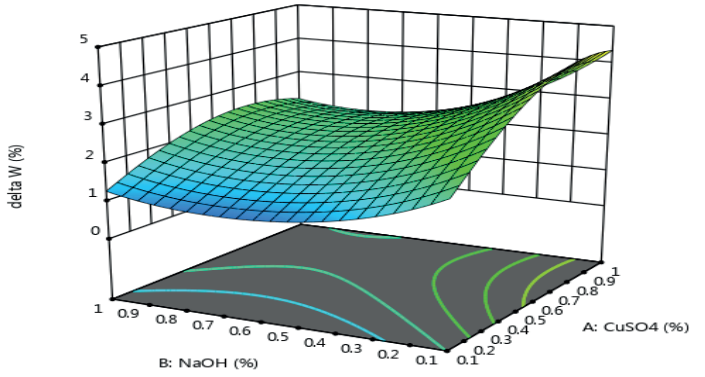

Design-Expert( (\$) Software

Factor Coding: Actual

Original Scale

delta $\mathbf{W}(\%)$

$0.26 \square 5.78$

$\mathrm{X} 1=\mathrm{A}: \mathrm{CuSO} 4$

$\mathrm{X}_{2}=\mathrm{B}: \mathrm{NaOH}$

Actual Factors

C: Time $=120$

$\mathrm{D}: \mathrm{CTAB}=0.35$

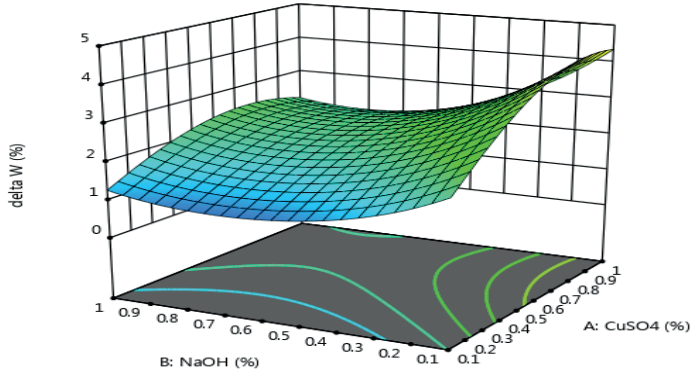

f

Design-Expert( (i) Software

Factor Coding: Actual

Original Scale

delta W (\%)

$0.26 \square 5.78$

$\mathrm{X} 1=\mathrm{A}: \mathrm{CuSO} 4$

$\times 2=\mathrm{B}: \mathrm{NaOH}$

Actual Factors

C: Time $=120$

$\mathrm{D}: \mathrm{CTAB}=0.35$

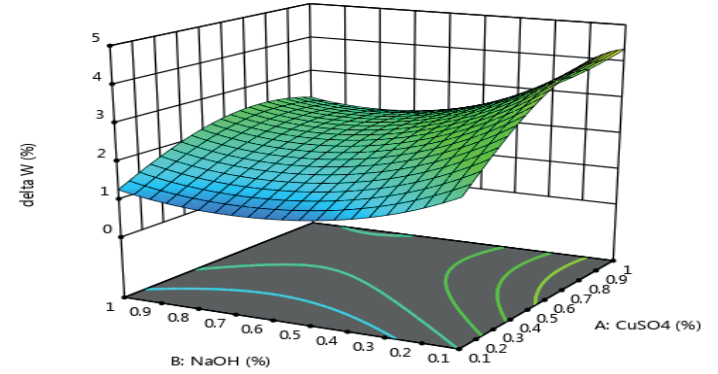

g

Fig2. 3D response surface for weight changes(fig a-g) 
Figure (3) shows the 3D response surface for the desirability conditions. These conditions were adjusted based on the lowest amount of $\mathrm{CTAB}$ and the maximum operating time.

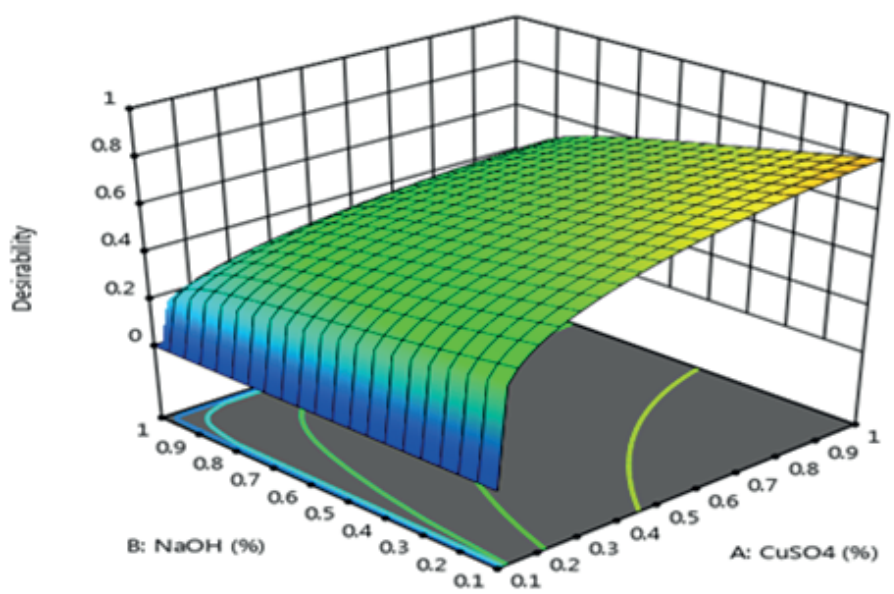

Fig3. Three-dimensional surface of response to achieve desirable conditions

According to the surface treatment performed on the fabric, a sample with more weight changes and less color changes in the surface of the fabric is selected as the optimum sample.
For statistical analysis of the operation, we used analysis of variance (ANOVA) based on weight and color variations. Color and weight variations are shown in Table (2) based on ANOVA analysis.

Table 2. ANOVA test for CTAB treated samples

\begin{tabular}{ccccccc}
\hline & Sum of Squares & df & Mean Square & F & Sig. & \\
\hline weight & Between Groups & 64.906 & 24 & 2.704 & 2.121 & 0.013 \\
changes $(\Delta \mathrm{W})$ & Within Groups & 63.756 & 50 & 1.275 & & \\
& Total & 128.662 & 74 & & & \\
color changes $(\Delta \mathrm{E})$ & Between Groups & 9134.647 & 24 & 380.61 & 1.896 & 0.028 \\
& Within Groups & 10035.189 & 50 & 200.704 & & \\
& Total & 19169.836 & 74 & & & \\
\hline
\end{tabular}

The obtained models based on the different copper sulfate and sodium hydroxide in the presence of CTAB are reported in equations (1) and (2).

$\Delta \mathbf{E}^{\wedge} \mathbf{0 . 1 7}=2.11-0.59 * \mathrm{CuSO}_{4}-0.16 * \mathrm{NaOH}-0.004 *$ Time $-0.18 * \mathrm{CTAB}-0.029 * \mathrm{CuSO}_{4} * \mathrm{NaOH}+0.003 * \mathrm{CuSO}_{4} *$ Time +0.52 $* \mathrm{CuSO}_{4} * \mathrm{CTAB}+0.002 * \mathrm{NaOH} *$ Time $+0.10 * \mathrm{NaOH} * \mathrm{CTAB}-0.002 * \mathrm{Tim}^{*}{ }^{*} \mathrm{CTAB}$

$\Delta \mathbf{W}^{\wedge} \mathbf{0 . 6 1}=1.21+1.12 * \mathrm{CuSO}_{4}-1.90 * \mathrm{NaOH}+0.028 * \mathrm{Time}^{-0.65} * \mathrm{CTAB}-0.48 * \mathrm{CuSO}_{4} * \mathrm{NaOH}+0.013 * \mathrm{CuSO}_{4} * \mathrm{Ti}-$ me-0.089 ${ }^{*} \mathrm{CuSO}_{4}{ }^{*} \mathrm{CTAB}-0.006 * \mathrm{NaOH}^{*} \mathrm{Time}+0.58 * \mathrm{NaOH}^{*} \mathrm{CTAB}-0.0027 * \mathrm{Time}^{*} \mathrm{CTAB}-1.48 * \mathrm{CuSO}_{4}{ }^{2}+1.87 * \mathrm{Na}-$ $\mathrm{OH}^{2}-0.00019 *$ Time $^{2}+0.68 * \mathrm{CTAB}^{2}$

(2) 
Finally, an optimum specimen is the sample treated with $1 \%$ copper sulfate, $0.1 \%$ sodium hydroxide, 0.35 $\%$ CTAB at boiling point for $120 \mathrm{~min}$. At this stage, after determining the optimum sample. Subsequent experiments were performed to investigate the synthesis of nano-copper on the fabric.

To perform nano-copper synthesis studies, electron microscopy (SEM), x-ray diffraction (EDX), Fourier transform infrared spectroscopy (FTIR), X-ray diffraction (XRD) were used.

\section{METHODS}

\subsection{Synthesis of nano-copper in solution}

Possible reactions are (1-5) for the synthesis of nanocopper on a polyester fabric in an aqueous medium. CTAB can be reacted with copper sulfate and sodium hydroxide $(1,2)$.

According to reaction (3), due to the heat, copper hydroxide is first converted to copper oxide, and then through the formation of reducing agent in the environment through the reaction of alkali with the ester in polyester, to zero copper. This is shown in reaction (4).
$\begin{array}{ll}\mathrm{C}_{19} \mathrm{H}_{42} \mathrm{BrN}+\mathrm{CuSO}_{4} \longrightarrow \mathrm{BrSO}_{4}+\mathrm{C}_{19} \mathrm{H}_{42} \mathrm{NCu} \\ \mathrm{C}_{19} \mathrm{H}_{42} \mathrm{NCu}+2 \mathrm{NaOH} \longrightarrow \mathrm{Cu}(\mathrm{OH})_{2}+\mathrm{C}_{19} \mathrm{H}_{42} \mathrm{Na}_{2} \mathrm{~N} \\ \mathrm{Cu}(\mathrm{OH})_{2} \\ \mathrm{CuO}+\text { reducing groups } \longrightarrow \mathrm{CuO}+\mathrm{H}_{2} \mathrm{O} \\ \text { Heating } & \longrightarrow \mathrm{CuO}+\mathrm{H}_{2} \mathrm{O}\end{array}$
Moreover, the polyester fabric in the presence of alkali is decomposed and turns into negative temple, where positive sodium ions can react with them according to
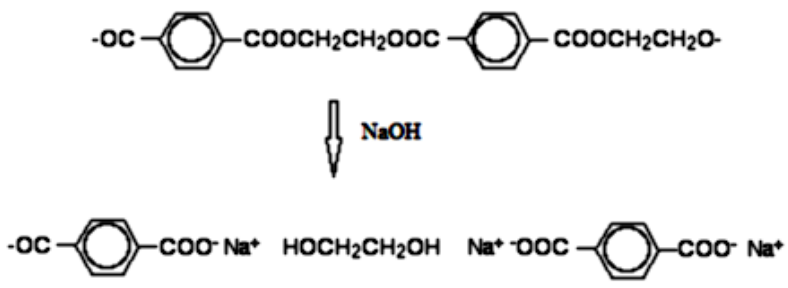

reaction (5), and in the case of presence of positive copper replaces sodium ions.
Figure (4) shows nano-copper synthesis using copper sulfate, sodium hydroxide and CTAB in aqueous solution. Due to the simultaneous presence of the fabrics in the solution, these materials form bonds with active groups in the polyesters physically and chemically [11], [12].

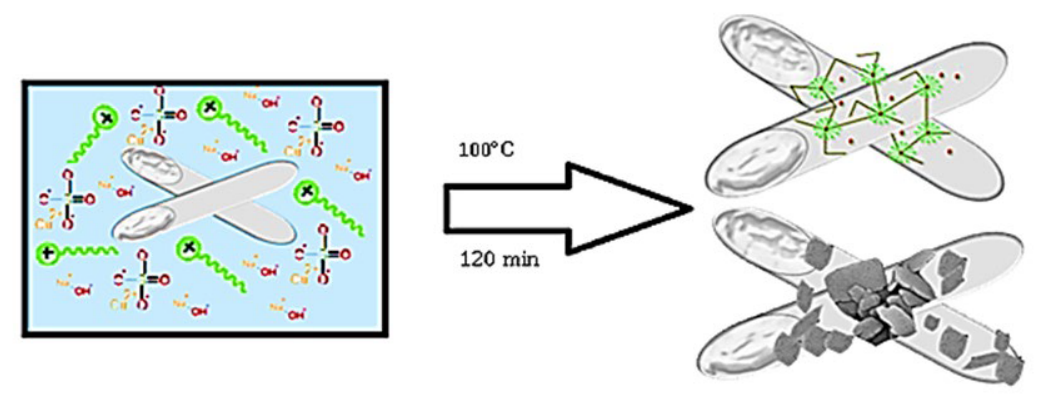

Fig4. Schematic of nano-copper synthesis on polyester fabric using CTAB 
Therefore, based on chemical reaction (1-5), mainly using CTAB can cause different roles in the process of synthesis of nano-copper. It can act as controlling the particle size, reducing agent and preventing agglomeration and oxidation or bonding agents to the fiber and the fabric surface, depending on the conditions. This can carry out one or more of these tasks.

\subsection{Surface reflection (K/S), $\Delta \mathrm{E}$}

The amount of $\mathrm{K} / \mathrm{S}$ was investigated at a wavelength of $600 \mathrm{~nm}$ and reported to be about 0.12. Also, the $\Delta \mathrm{E}$ is reported at about 13.5 . This shows very light color of the treated fabric that can be dyed in the following step.

\subsection{Studying the chemical structure based on FTIR spectrum}

In this experiment, the chemical structure of the raw polyester fabric and the nano-copper modified polyester fabric were examined. The results of changes in the size of the peaks or their displacement in FTIR spectroscopy show that it can be attributed to the effect of corrective actions on the chemical structure of the fabric. The FTIR spectra of samples on the fabric are shown in Figure (5).
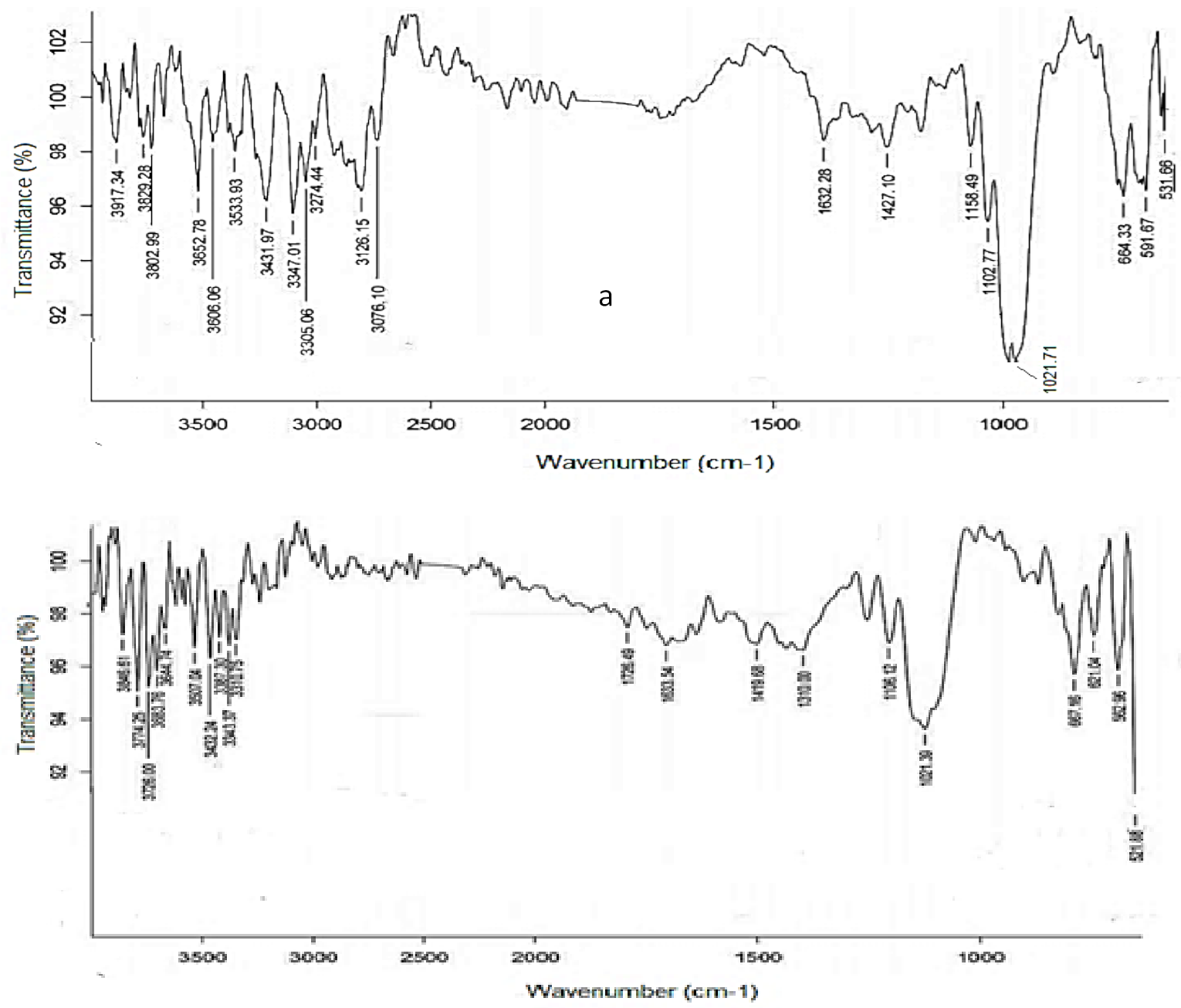

Fig5. FTIR spectrum related to a) raw and b) modified polyester fabrics with CTAB 
These peaks or changes represent additional operations effect on the fabric chemical structure.

\subsection{X-ray diffraction (XRD)}

The crystal structure of the sample modified by XRD using electronic ray bombardment $20-40 \mathrm{KeV}$ and wavelength $(\gamma=0.154 \mathrm{~nm})$ in the range of 2 Theta from 10 to 100 degrees angle and at every step for 0.02 degrees has been scanned.
According to the standard JCPDS Copper: 04-0836, 2theta of nano-copper is specified in three values of $43.297,50.433$ and 74.420 that are mainly related to the Miller indices (hkl) for the peaks (111), (200) and (220).

The intensity and width of the peaks depend on the size of nanoparticles and their values [16]. The existences of prominent peaks related to nanocopper were shown near the standard range of the device. The results are reported in Figure (6).

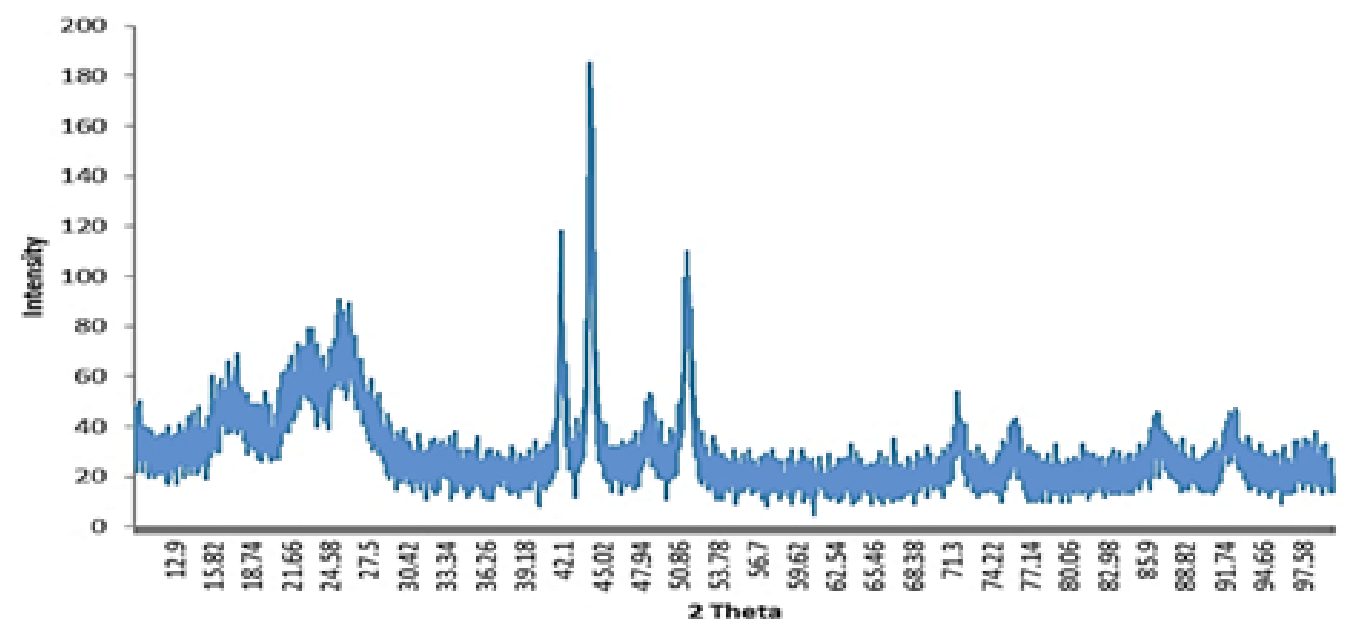

Fig6. X-ray diffraction related to the finishing sample using CTAB

\subsection{Electron microscope analysis (SEM / EDX)}

The nanoparticles on the surface of the fabric can be seen by using SEM images. Nanoparticle size can be measured using more magnification and their shape can be observed in different forms on the fabric [17].
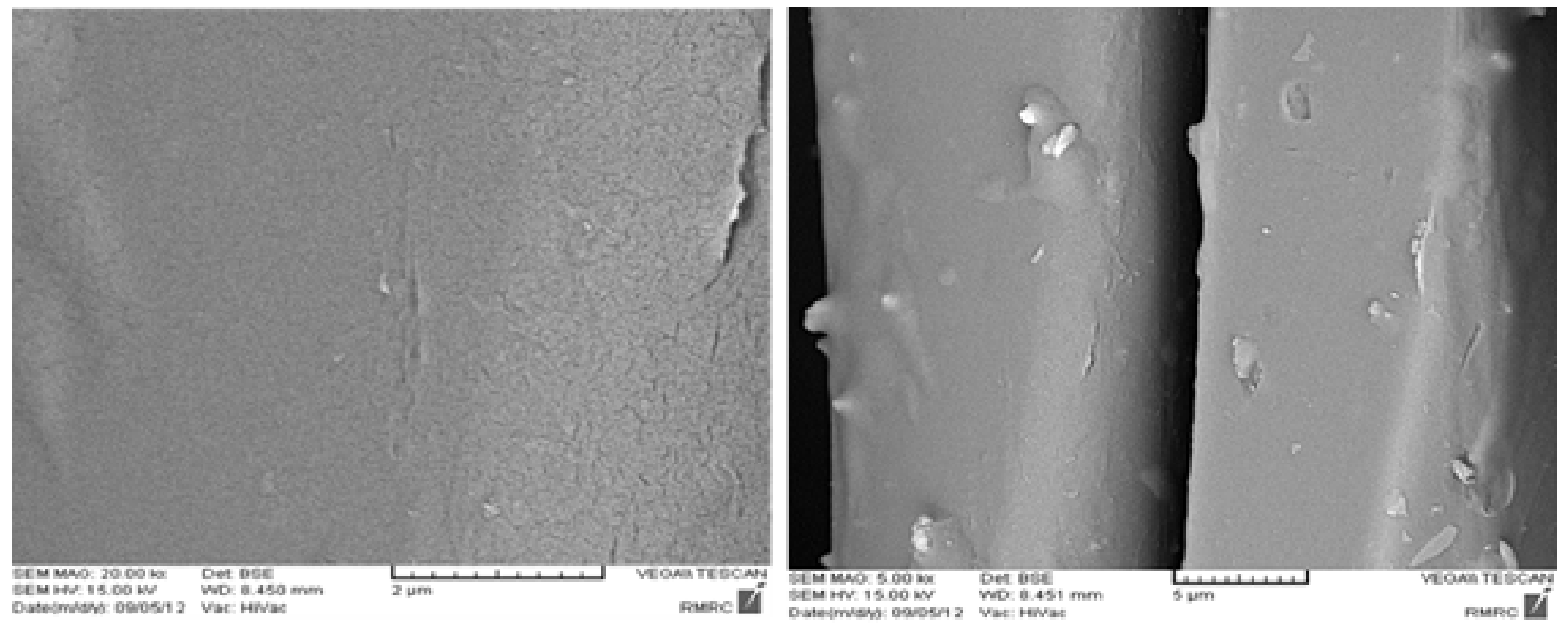

Fig. 7. Raw sample image at a magnification of 20000 and 5000
In Figure (7), SEM image of the raw sample is shown where the fiber surface before treatment is smooth and uniform. Based on electron microscopic images, copper nanoparticles in Figure (8) related to the treated sample. 
Figure (8) shows the synthesis of nano copper deposed on the fabric surface. The important point is the form of copper nanoparticles synthesized
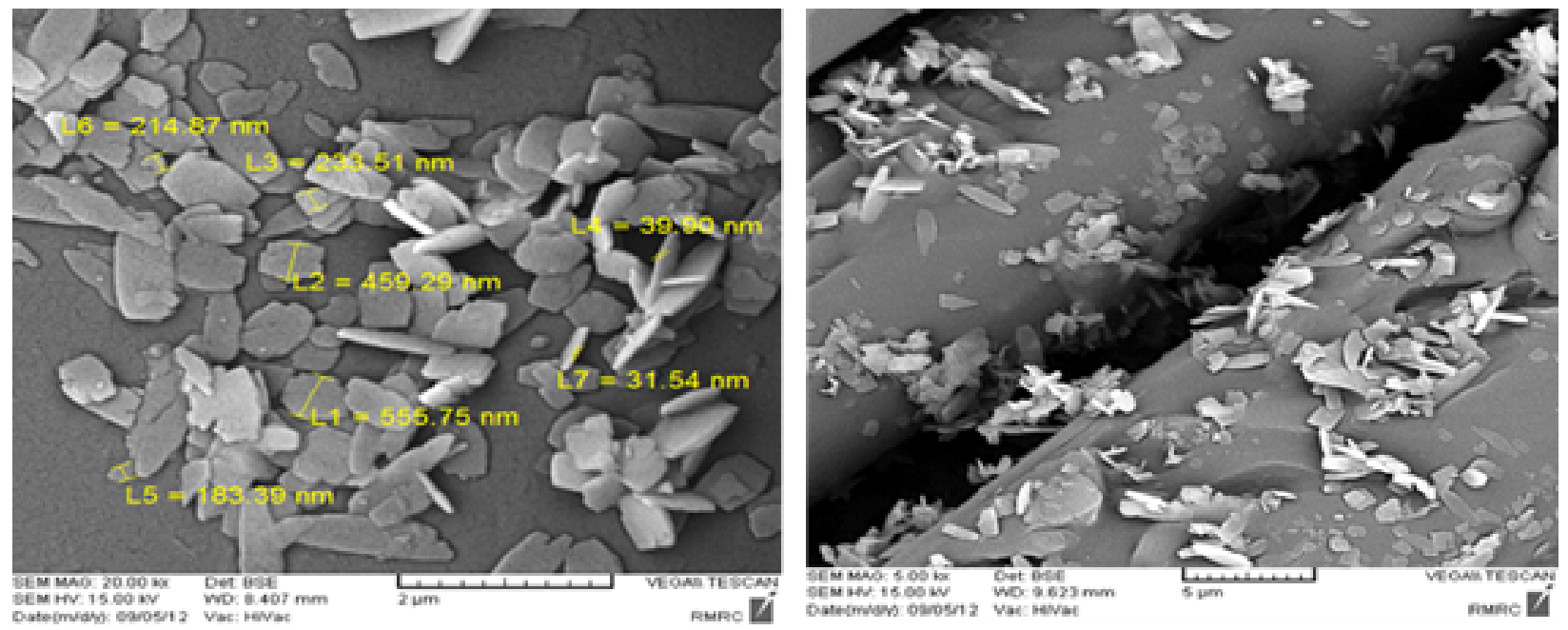

Fig. 8. Surface of the treated sample with copper sulphate and CTAB with a magnification of 20000 and 5000

The reason for the formation of nanoplates could be reaction of copper ions with CTAB and sodium hydroxide.

\subsection{Analysis of the EDX patterns}

The combination of copper nanoparticles was studied with X-rays and analyzed by EDX. This spectrum shows a number of sharp peaks that correspond to the various elements on the fabric surface [18], [19]. In Figure (9), the elements of copper, carbon and oxygen are shown based on the filter.

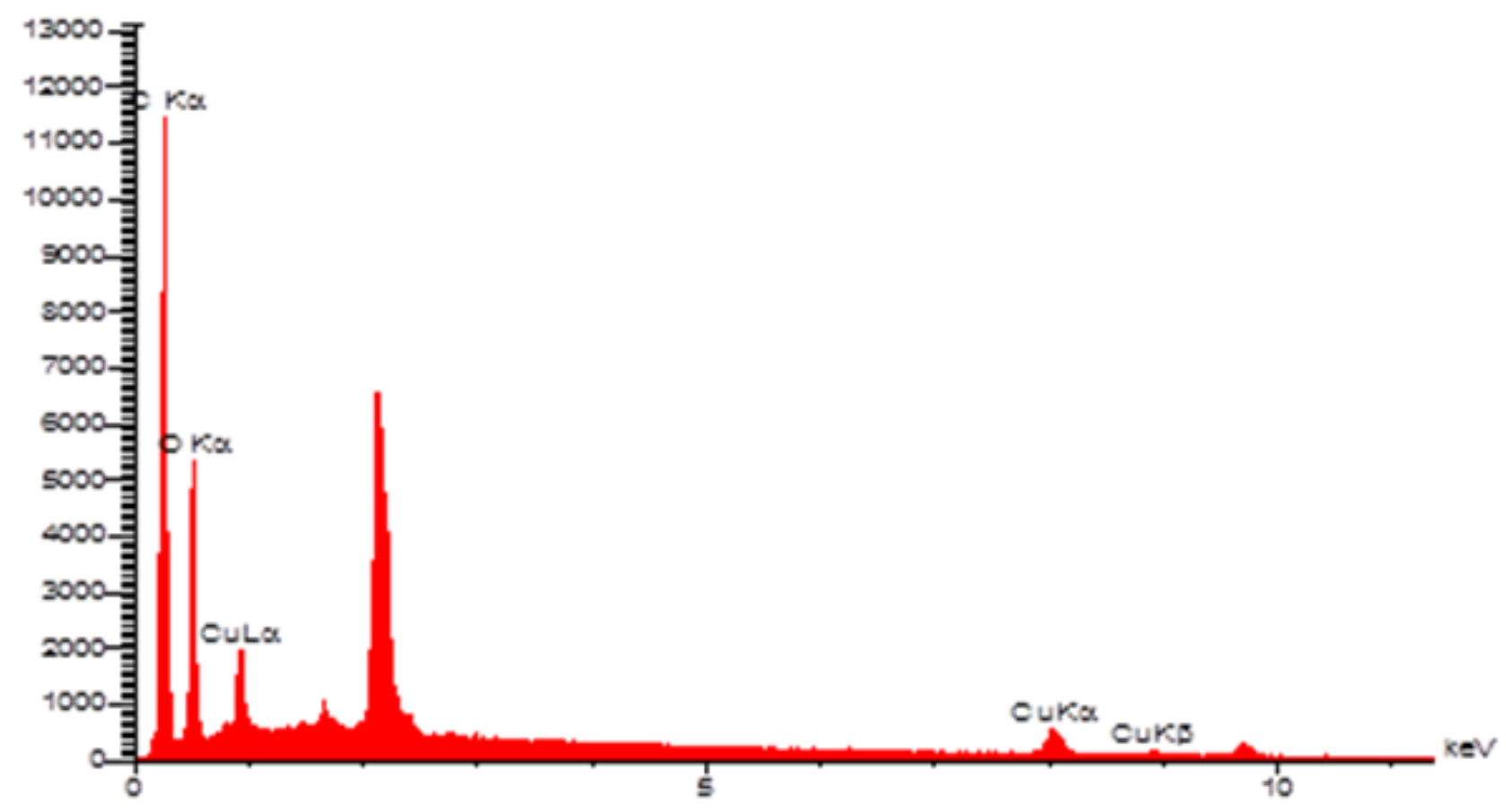

Fig9. EDX analysis of the treated sample with CTAB 
According to EDX analysis, it can specify the existence of copper on the treated sample. Copper content on the surface of the fabric based on EDX analysis for the finished sample is $0.16 \%$.

\section{CONCLUSION}

This study shows the synthesis of nano-copper and surface modification of polyester fabric with using copper sulphate in the presence of sodium hydroxide and CTAB in one step. CTAB has a different role in the synthesis process of nanocopper on polyester fabric including controlling the nanoparticle size, reducing agent, preventing the oxidation acting as capping agent. Moreover, the placement of synthesized copper nanoparticles with different sheets with very low thickness occurred on polyester fabric. SEM images showed the nanocopper synthesis on polyester fabric as plates in length of 150 to $600 \mathrm{~nm}$ and thickness about 30 nm. Nanosheets in different shapes of roughly rectangle can be due to the molecular weight of the compounds created in this research.

\section{REFERENCES}

1. E. Halevas and A. Pantazaki, "Copper Nanoparticles as Therapeutic Anticancer Agents," vol. 2, no. 1, p. 119, 2018.

2. S. Chandra, A. Kumar, and P. K. Tomar, "Synthesis and characterization of copper nanoparticles by reducing agent," J. Saudi Chem. Soc., vol. 18, no. 2, pp. 149-153, Apr. 2014.

3. S. A. Patil, C.-H. Ryu, and H.-S. Kim, "Synthesis and Characterization of Copper Nanoparticles (Cu-Nps) using Rongalite as Reducing Agent and Photonic Sintering of Cu-Nps Ink for Printed Electronics," Int. J. Precis. Eng. Manuf. Technol., vol. 5, no. 2, pp. 239-245, Apr. 2018.

4. Q. ZHANG, Z. YANG, B. DING, X. LAN, and Y. GUO, "Preparation of copper nanoparticles by chemical reduction method using potassium borohydride," Trans. Nonferrous Met. Soc. China, vol. 20, pp. s240-s244, May 2010.

5. A. Umer, S. Naveed, N. Ramzan, M. S. Rafique, and M. Imran, "A green method for the synthesis of copper nanoparticles using l-ascorbic acid," Rev. Mater., 2014.
6. S. Shahidi, J. Wiener, and M. Ghoranneviss, "Surface Modification Methods for Improving the Dyeability of Textile Fabrics," in EcoFriendly Textile Dyeing and Finishing, InTech, 2013.

7. A. B. Rezaie and M. Montazer, "Polyester modification through synthesis of copper nanoparticles in presence of triethanolamine optimized with response surface methodology," Fibers Polym., vol. 18, no. 3, pp. 434-444, Mar. 2017.

8. M. Abdulla-Al-Mamun, Y. Kusumoto, and M. Muruganandham, "Simple new synthesis of copper nanoparticles in water/acetonitrile mixed solvent and their characterization," Mater. Lett., vol. 63, no. 23, pp. 2007-2009, Sep. 2009.

9. B. H. Chattopadhyay, Patel, "Effect of nanosized colloidal copper on cotton fabric," J. Eng. Fabr. Fibers, vol. 5, no. 3, pp. 1-6, 2010.

10. M. M. Jolaei, M. Montazer, A. Rashidi, and M. B. Moghadam, "Usage of alkaline glucose for Synthesis Copper Nano particle on Polyester Fabric," Ciência e Nat., vol. 37, p. 63, Dec. 2015.

11. M. Montazer and M. M. Jolaei, "Novel spacer three-dimensional polyester fabric with $\beta$ -cyclodextrin and butane tetra carboxylic acid," J. Text. Inst., vol. 101, no. 2, pp. 165-172, Feb. 2010.

12. M. Montazer and M. M. Jolaei, 'Î2-Cyclodextrin stabilized on three-dimensional polyester fabric with different crosslinking agents," J. Appl. Polym. Sci., vol. 116, no. 1, pp. 210-217, Apr. 2010.

13. M. M. Jolaei, "Improvement of 3D Polyester Fabric Surface with Nano Beta-Cyclodextrin and Hydrophilic Silicone and Microemulsion Softeners," International Journal of Advanced Biotechnology and Research (IJBR), vol. 7. pp. 1314-1324.

14. H. R. Hong, J. Kim, and C. H. Park, "Facile fabrication of multifunctional fabrics: use of copper and silver nanoparticles for antibacterial, superhydrophobic, conductive fabrics," RSC Adv., vol. 8, no. 73, pp. 41782-41794, 2018.

15. M. M. Jolaei, M. Montazer, A. S. Rashidi, and M. B. Moghadam, "Green synthesis copper nano particles for increasing antibacterial properties of polyester fabrics," Res. J. Pharm. Biol. Chem. Sci., 2016. 
16. J. Lim, S. P. Yeap, H. X. Che, and S. C. Low, "Characterization of magnetic nanoparticle by dynamic light scattering," Nanoscale Res. Lett., vol. 8, no. 1, p. 381, Sep. 2013.

17. A. Nasirian, "Synthesis and characterization of $\{\mathrm{Cu}\}$ nanoparticles and studying of their catalytic properties," Int. J. Nano Dimens., vol. 2, no. 3, pp. 159-164, Mar. 2012.
18. H. Zhao, T. Liang, and B. Liu, "Synthesis and properties of copper conductive adhesives modified by SiO2 nanoparticles," Int. J. Adhes. Adhes., 2007.

19. X. Song, S. Sun, W. Zhang, and Z. Yin, "A method for the synthesis of spherical copper nanoparticles in the organic phase," J. Colloid Interface Sci., 2004. 